\title{
Lysosomal Biogenesis and Implications for Hydroxychloroquine Disposition ${ }^{\mathbb{1}}$
}

\author{
Keagan P. Collins, Sandra Witta, Jonathan W. Coy, Yi Pang, and Daniel L. Gustafson \\ Colorado State University, School of Biomedical Engineering (K.P.C., S.W., D.L.G.) and Department of Clinical Sciences (D.L.G., \\ J.W.C.), Colorado State University, Fort Collins, Colorado; University of Colorado Cancer Center, Anschutz Medical Campus, \\ Aurora, Colorado (D.L.G.); and University of Akron, Department of Chemistry, Akron, Ohio (Y.P.)
}

Received August 28, 2020; accepted October 26, 2020

\begin{abstract}
Lysosomes act as a cellular drug sink for weakly basic, lipophilic (lysosomotropic) xenobiotics, with many instances of lysosomal trapping associated with multiple drug resistance. Lysosomotropic agents have also been shown to activate master lysosomal biogenesis transcription factor EB (TFEB) and ultimately lysosomal biogenesis. We investigated the role of lysosomal biogenesis in the disposition of hydroxychloroquine (HCQ), a hallmark lysosomotropic agent, and observed that modulating the lysosomal volume of human breast cancer cell lines can account for differences in disposition of $\mathrm{HCQ}$. Through use of an in vitro pharmacokinetic (PK) model, we characterized total cellular uptake of $\mathrm{HCQ}$ within the duration of static equilibrium (1 hour), as well as extended exposure to HCQ that is subject to dynamic equilibrium ( $>1$ hour), wherein $\mathrm{HCQ}$ increases the size of the lysosomal compartment through swelling and TFEBinduced lysosomal biogenesis. In addition, we observe that pretreatment of cell lines with TFEB-activating agent Torin1 contributed to an increase of whole-cell HCQ concentrations by
\end{abstract}

1.4- to 1.6-fold, which were also characterized by the in vitro PK model. This investigation into the role of lysosomal volume dynamics in lysosomotropic drug disposition, including the ability of $\mathrm{HCQ}$ to modify its own disposition, advances our understanding of how chemically similar agents may distribute on the cellular level and examines a key area of lysosomalmediated multiple drug resistance and drug-drug interaction.

\section{SIGNIFICANCE STATEMENT}

Hydroxychloroquine is able to modulate its own cellular pharmacokinetic uptake by increasing the cellular lysosomal volume fraction through activation of lysosomal biogenesis master transcription factor EB and through lysosomal swelling. This concept can be applied to many other lysosomotropic drugs that activate transcription factor $E B$, such as doxorubicin and other tyrosine kinase inhibitor drugs, as these drugs may actively increase their own sequestration within the lysosome to further exacerbate multiple drug resistance and lead to potential acquired resistance.

\section{Introduction}

Hydroxychloroquine (HCQ) has been investigated in over 50 cancer clinical trials and countless preclinical studies through the past decade as an additive compound to primary chemotherapy treatment. Mechanistically, HCQ acts to inhibit autophagy, which has been identified as a mechanism of tumor cell resistance to chemotherapy and a key pathway to survival within the tumor microenvironment. Although nextgeneration autophagy inhibitors are in preclinical stages of development, HCQ remains the only clinically approved

This work was supported by National Institutes of Health National Cancer Institute [Grant R01CA190170] (Therapeutic Targeting of AutophagyDependent Cancer) and [Grant P30CA046934](University of Colorado Cancer Center Support Grant, Pharmacology Shared Resource), by Department of Defense Congressionally Directed Medical Research Program Breast Cancer Research Program [Grant BC130103P1] (Identifying and Targeting Autophagy Dependence to Eliminate Metastatic Breast Cancer), and by the Shipley University Chair in Comparative Oncology [DL Gustafson].

The authors declare that no conflict of interest exists.

https://doi.org/10.1124/jpet.120.000309.

S This article has supplemental material available at jpet.aspetjournals.org. autophagy inhibitor. Overall, HCQ is generally effective and safe as a treatment but suffers from various pitfalls within the pharmacologic space-specifically in terms of predictability of patient likelihood of response and reliable pharmacokinetic (PK) and pharmacodynamic (PD) metrics. There is a general disconnect between HCQ PK in the tumor and blood, which makes it challenging to be confident that target concentrations of HCQ are achieved within the tumor when optimizing dosing strategies (Barnard et al., 2014). Characterization of HCQ PK properties on a cellular level may provide insight toward tumor-specific factors that would promote this disconnect.

Cellular uptake of HCQ is driven primarily through lysosomal ion trapping due to the weakly basic and lipophilic properties that characterize it as a hallmark lysosomotropic agent. HCQ readily diffuses through neutral spaces but becomes diprotonated in the lysosome, whose acidic environment facilitates accumulation. This results in concentrations significantly higher than other compartments within the cell (Duvvuri and Krise, 2005). Key mechanisms contributing to pharmacokinetics have been considered and consolidated into

ABBREVIATIONS: AUC, area under the curve; Cl, confidence interval; Ctrl, control; CQ, chloroquine; Cy3, Cyanine; DAPI, 4',6-diamidino-2phenylindole; GSEA, gene set enrichment analysis; $\mathrm{HCQ}$, hydroxychloroquine; MAPE, median absolute performance error; MN, monensin; MPE,

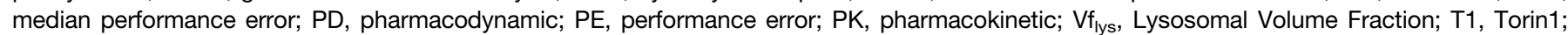
TFEB, transcription factor EB; TKI, tyrosine kinase inhibitor. 
in vitro mathematical models to describe cellular uptake of compounds with similar physicochemical properties to HCQnamely, weakly basic and lipophilic (Ishizaki et al., 2000; Trapp et al., 2008; Kornhuber et al., 2010; Schmitt et al., 2019). These models assume the cell behaves as a static system of compartments, but recent evidence suggests that lysosomotropic compounds induce changes in the lysosome itself.

Lysosomes swell in response to chloroquine (CQ) exposure, which is mechanistically and pharmacokinetically similar to HCQ. Near clinically relevant doses of CQ have been observed to cause a 3-fold increase in relative lysosomal volume after multiple days of exposure to the drug (King et al., 2016), and recruitment of galectin-3 to sites on these swollen lysosomes, indicating membrane damage (Gallagher et al., 2017). In addition to an increase in lysosomal size, an increase in lysosomal number has also been reported in response to a panel of lysosomotropic agents, including CQ, over the course of 4 and 24 hours (Lu et al., 2017). This increase in lysosomal number was linked to an increase in nuclear translocation of master lysosomal biogenesis transcription factors TFEB, transcription factor binding to IGHM enhancer 3 (TFE3), and melanocyte inducing transcription factor (MITF). TFEB activation of the coordinated lysosomal expression and regulation (CLEAR) network of genes has been described as the master regulation system of lysosomal biogenesis (Settembre et al., 2011, 2012). Lysosomotropic agents cause activation of this network by inducing lysosomal stress, and it has been shown to activate with as little as 90 minutes of exposure to CQ (Zhitomirsky et al., 2018). The results of lysosomal swelling and TFEB activation by lysosomotropic agents may be linked to their long-term tissue PK. Extended dosing of HCQ is characterized by a consistent increase in HCQ concentrations in rat tissue over the course of 3 months of daily dosing. In some tissues (spleen, kidney, liver, heart) HCQ concentrations continued to increase out to the latest time point measured (at 7 months) (McChesney, 1983). This long-term PK profile is noted by McChesney to be similar to other lysosomotropic drugs, such as chlorpromazine.

Because of the frequent, high doses of HCQ used in cancer clinical trials, we characterized this lysosomal adaptation response on a cellular level and investigated the extent to which it may influence cellular disposition of the drug. The data described herein suggest that baseline cell uptake of HCQ is associated with different baseline lysosomal profiles between cell lines, which were integrated into a lysosome cellular PK mathematical model (Trapp et al., 2008; Kornhuber et al., 2010) to accurately model whole-cell HCQ PK to chemical kinetic equilibrium. Small but consistent increases in whole-cell uptake of HCQ over an extended time period were also observed and were attributed to an increasing volume of the lysosomal compartment. The in vitro PK profile of HCQ was thus broken up into a short-term kinetic equilibrium portion, within 1 hour of exposure, and a long-term dynamic portion, out to 24 hours of exposure, for all cell lines. These data were used in conjunction with observed differences in baseline lysosomal profiles to further characterize the cellular PK of HCQ by accounting for a dynamic lysosomal compartment.

\section{Materials and Methods}

Cell Lines. Human breast cancer cell lines, including MDA-MB231, MDA-MB-468, T47D, and MCF7 human breast cancer cell lines, were purchased from Cell Culture Services at University of Colorado Anschutz Medical Campus (Aurora, CO). For cell pharmacokinetic experiments, all cell lines were stably transfected with Incucyte NucLight Red Lentivirus (4476; Sartorius, Gottingen, Germany) prior to experimentation.

Cell lines were cultured in Dulbecco's modified essential medium (10-017-CV; Corning) supplemented with 10\% fetal bovine serum (PSFB3; Peak Serum), 1\% penicillin/streptomycin (30-002-CI; Fisher, Hampton, NH), and $1 \%$ sodium pyruvate $\left(25-000-\mathrm{CI}\right.$; Fisher) at $37^{\circ} \mathrm{C}$ and $5 \% \mathrm{CO}_{2}$ in a humidified incubator.

Chemicals and Reagents. HCQ sulfate was purchased from Sigma-Aldrich (St. Louis, MO). Monensin (MN) was purchased from VWR (Radnor, PA). Torin1 (T1) was purchased from Fisher. Hoechst $3334220 \mathrm{mM}$ solution (62249) and CellLight Lysosomes-GFP, BacMam 2.0 (C10507) were purchased from ThermoFisher (Waltham, MA). pyrene-benzothiazolium dye 1b (ETP), a pH-insensitive lysosomal lumen dye applicable for live and fixed cell imaging, was received as a gift from Dr. Yi Pang at the University of Akron (Abeywickrama et al., 2019).

Cell Uptake Experiments. Four human breast cancer cell lines were used to perform experiments. The first two, MBA-MB-231 and MDA-MB-468, are mechanistically sensitive to autophagy inhibition through gene knockdown (Maycotte et al., 2014; Towers et al., 2019) and pharmacologically sensitive to hydroxychloroquine. The second two, MCF7 and T47D, are insensitive to both methods (Maycotte et al., 2014).

Cell lines were cultured in standard Dulbecco's Modified Eagle Medium (DMEM) supplemented with 10\% FBS, 1\% penicillin streptomycin (PS), and $1 \%$ sodium pyruvate in an incubator at $37^{\circ} \mathrm{C}$ and $5 \% \mathrm{CO}_{2}$. T47D and MCF7 cell lines required supplementation of $8 \mu \mathrm{g} / \mathrm{ml}$ of insulin to maintain growth. For acidic media experiments with MDA-MB-231 cells, Dulbecco's Modified Eagle Medium (DMEM) without sodium bicarbonate was purchased, and bicarbonate was added to achieve an incubator-conditioned media $\mathrm{pH}$. Culture media buffers other than sodium bicarbonate, such as HEPES, were purposely omitted, as HEPES has been shown to activate the micropthalmia/transcription factor $\mathrm{E}$ (MiT/TFE) network, resulting in lysosomal biogenesis (Tol et al., 2018).

Cell lines were plated, allowed to adhere overnight, and treated at roughly $70 \%$ confluence. All cell lines stably expressed NucLight Red, and cell counts were measured by counting red fluorescent nuclei on an Incucyte ZOOM (Sartorius) prior to treatment with drug. For 4- and 24-hour time points, cells were counted immediately prior to harvesting, as the doubling time for these cell lines is between 16 and 20 hours. Experiments were not extended past 24 hours to avoid cell line growth inhibition in response to HCQ. For treatment, culture media was aspirated and replaced with fresh, incubator-conditioned media containing $10 \mu \mathrm{M} \mathrm{HCQ}$ and placed in a $37^{\circ} \mathrm{C}$ incubator until the designated time point. At the time point, cell media containing HCQ were aspirated, and cells were washed with Hank's balanced salt solution (HBSS) and then trypsinized for 5 minutes. Trypsinized cells were washed off the plate with additional culture media at a ratio of 4:1 media:trypsin. Cells were then pelleted at $1600 \mathrm{rpm}$ for 5 minutes, media were carefully aspirated, and the cell pellet was reconstituted in MilliQ-water and frozen until liquid chromatography with tandem mass spectrometry (LC/MS-MS) extraction and analysis.

(LC/MS-MS) analysis follows methods described in Barnard et al. (2014); briefly, cell pellets were vortexed and lysed for 10 minutes in a sonication water bath. Acetonitrile was then added to the cell pellets to yield a final solution of $50 \%$ acetonitrile $+50 \%$ MilliQ- $\mathrm{H}_{2} \mathrm{O}$. Cells were vortexed for 10 minutes and then centrifuged at 13,300 rpm for 10 minutes. Supernatant was transferred to polypropylene inserts. CQ was used as an internal standard. Concentrations of supernatant obtained on LC/MS-MS were normalized to the cell counts obtained for each sample from an Incucyte ZOOM, and samples were then normalized to cell volume, obtained for each cell line from suspended cell diameter measured on a Countess system $(n=4)$, to obtain HCQ concentration (micromolars) in each cell. 
For experiments with MN, cells were pretreated with $25 \mu \mathrm{M} M N$ for 30 minutes prior to adding HCQ. After 30 minutes, $10 \mu \mathrm{M}$ HCQ was added for the duration of the 1-hour exposure. For experiments with T1, cells were pretreated with $250 \mathrm{nM}$ Torin 1 for 16 hours prior to adding HCQ. All cell uptake experiments were performed at least in triplicate.

Lysosomal Imaging. For all imaging experiments, cells were plated at 10,000 cells per well on an eight-well glass chamber slide (C8-1.5H-N; Cellvis) and allowed to adhere overnight.

To determine lysosomal volume, live cells were treated with $1 \mu \mathrm{M}$ ETP (Abeywickrama et al., 2019) and incubated for 30 minutes. Excess dye was removed by washing twice with warm media and incubated with $10 \mu \mathrm{M}$ of Hoescht 33342 for 10 minutes. Cells were then washed twice with PBS and fixed for 10 minutes in $2 \%$ paraformaldehyde (PFA). PFA was washed off twice with PBS, and cells were imaged on an Olympus IX83 microscope with a Plapon $60 \times$ objective, numerical aperture $(\mathrm{NA})=1.42$, on Cy3 and DAPI confocal channels. In total, 43 z-stacks of $0.24-\mu \mathrm{m}$ step size were captured on Cy3 and DAPI channels. Lysosomal volume fraction of cells was determined using an in-house image processing pipeline, which is described briefly as follows: raw confocal image stacks were converted to tagged image file format (TIF) files and imported into Python version 3.7.6. A reference image was created by compressing the stack by its maximum pixel intensity. Each slice of each image stack was analyzed to determine the peak signal-to-noise ratio relative to the reference image using the sewar module (https://pypi.org/project/sewar/). A comma separated value (CSV) file containing the top 10 peak signal-to-noise ratio slices for each image stack was generated and imported into a FIJI macro that calculated the Cy3 (lysosome) area for each raw VSI image slice based on an algebraic threshold of mean intensity. The area for each slice was multiplied by the step size $(0.24 \mu \mathrm{m})$ to generate a volume, and then volumes from the 10 slices per image stack were summed to generate a lysosomal volume for the image. The lysosomal volume was normalized to the cell count of each image, and the cell count was normalized to the experimentally determined cell volume for each cell line to generate a lysosomal volume fraction per cell. In total, 5 to 10 images were obtained for each experimental replicate, and lysosomal volume fraction is presented as the mean \pm S.D. of each replicate $(n=3)$ for each cell line. Imaging of cell lines for GFP-lysosomal associated membrane protein 1 (LAMP1) expression followed the same imaging process, except cells were incubated with $1 \mu$ l of the baculovirus overnight prior to treatment with HCQ.

For drug treatment conditions, single plane images were captured at $20 \times$ using the same microscope but an Uplsapo $20 \times$ objective, numerical aperture (NA) $=0.75$, on $\mathrm{Cy} 3$ and DAPI light-emitting diode (LED) channels. To determine a change in lysosome content of the cell, cells were treated with the same drug treatment conditions as described in the Cell Uptake Experiments section. Chamber slides were prepared in the same way as described above. Images were quantitated using a FIJI macro with an algebraic threshold of the mean intensity, and data were calculated as Cy3 area/DAPI area. Roughly 10 images were obtained for each experimental replicate, and data are reported as the mean \pm S.D. of each replicate $(n=4)$. Statistical significance was calculated as a one-way ANOVA with Dunnett's multiple comparisons test relative to the control, with significance as $P<0.05$.

TFEB Activation. TFEB activation by HCQ was determined with two different kinds of methods, including gene set enrichment analysis (GSEA) of the TFEB-regulated Coordinated Lysosomal Expression and Regulation (CLEAR) network and through a transcription factor EH-EB activity assay (TFEH-TFEB; RayBiotech). Gene expression microarray data were prepared for all four human breast cancer (hBC) lines following cell culture preparation steps as outlined in the Cell Uptake Experiments section. Cell lines were treated with $20 \mu \mathrm{M}$ HCQ for 24 hours prior to harvesting for gene expression preparation in triplicate. Prepared cell extraction homogenate was run on an HG_U133_Plus_2 chip. Cell intensity (CEL) file data were extracted and prepared for GSEA using the Affymetrix Bioconductor package in $\mathrm{R}$ and normalized using Robust Multi-array Average (RMA). Expression data were then analyzed using the GSEA 4.0.3 software provided by the Broad Institute. Gene sets from the four cell lines, treated with HCQ or vehicle, were analyzed in GSEA using a gene set representative of TFEB lysosome targets (Palmieri et al., 2011) and run in tandem with the Hallmarks of Cancer gene set data base version 7.1 provided in the GSEA software. The run was set for 1000 permutations following a "gene_set" permutation type. Data are reported as the normalized enrichment score (NES) value, and significance was determined by false discovery rate (FDR) q-value generated in the run. Raw cell intensity (CEL) files are available in the supplement. TFEB nuclear activation was performed in duplicate and was determined using the human TFEB transcription factor activity assay following the manufacturer's instructions. TFEB activity data are expressed as means \pm S.D. and analyzed by one-way ANOVA with Dunnett's multiple comparison test relative to control, with significance as $P<0.05$.

Cell Pharmacokinetic Mathematical Modeling and Simulation. To connect whole-cell HCQ PK with the perturbations observed in the lysosomal compartment, HCQ whole-cell uptake was simulated using a previously published base model of lysosomal uptake (Trapp et al., 2008; Kornhuber et al., 2010). Lysosomal volume fraction for each cell line was determined by imaging methods described above. Extracellular $\mathrm{pH}$ was determined by measuring the $\mathrm{pH}$ of incubator-equilibrated media in a $\mathrm{pH}$ meter (7.6) and then testing the change in cellular uptake of HCQ at the 1-hour time points in media of decreased $\mathrm{pH}$ (7.0). The influence of the lysosome and cytosolic $\mathrm{pH}$ was determined experimentally by pretreating MDA-MB-231 cells with MN, a lysosome-selective ionophore (Grinde, 1983), and simulating the experimental uptake of HCQ in cells after pretreatment. The model values of cytosolic $\mathrm{pH}$ of roughly 7.0 and lysosomal $\mathrm{pH}$ of 5.0 worked well with the cell lines used, which are similar to what has been previously reported for MCF7 cytosolic pH (Belhoussine et al., 1999) and MCF7 and MDA-MB-231 lysosomal pH values (Montcourrier et al., 1994). It is noted that the range of the $\mathrm{pH}$ values from different literature reports is quite broad, with lysosomal $\mathrm{pH}$ values reported around 4.2 for MDA-MB-231 cells (Ndolo et al., 2012), 4.5 for MCF7 and MDA-MB-231 cells (Wang et al., 2019), and 5.1 for MCF7 and MDA-MB-231 cells (Montcourrier et al., 1994). Cytosolic pH exhibited a similar trend from literature, with reported values around 6.9 for MCF7 (Belhoussine et al., 1999), 7.3 for MCF7 (Persi et al., 2018), and 7.4 for MDA-MB-231 and MCF7 (Wang et al., 2012). From the studies using multiple human breast cancer lines, which were also used in this work, the lysosomal and cytosolic $\mathrm{pH}$ were not very different between each cell line, so we chose to use fixed values for these parameters across all four cell line models. Additional factors that could potentially play a role in cellular uptake and clearance of HCQ, such as acid-phospholipid binding and endo/lysosomal turnover, were not investigated between cell lines.

Evaluation of Model Fit. Model fit versus experimental data were evaluated by comparing the experimental $\mathrm{AUC}_{0-1}$ hour or $\mathrm{AUC}_{0-24}$ hour to the simulated value for each cell line. Additionally, model accuracy was analyzed by calculating the performance error (PE), the median performance error (MPE\%) to investigate positive/negative bias, and the mean absolute performance error (MAPE\%) to determine overall model fit (Gustafsson et al., 1992). These calculations included all time points relevant to the parent figure.

Computer Simulation and Software. Intracellular PK model simulation was done in MATLAB version R2020a (Mathworks, Natick, MA), and the intracellular compartmental system was solved using the ode45 package. Image selection was done in Python version 3.7.6 using sewar and tqdm packages. Image analysis was done in ImageJ version $1.52 \mathrm{p}$ using methods described above. 


\section{Results}

Whole-Cell Uptake of HCQ Is Proportional to Basal Lysosomal Volume Fraction. The in vitro PK of HCQ was assessed in four human breast cancer (hBC) cell lines-MDA-MB-231, MDA-MB-468, T47D, and MCF7-by collecting total cell homogenate at $1,5,15,30,60,240$, and 1440 minutes after incubating with $10 \mu \mathrm{M}$ HCQ. This concentration of HCQ was chosen because it is within the upper range of clinically achievable plasma $\mathrm{C}_{\max }$ concentrations. HCQ concentration within the whole cell was determined by normalizing total HCQ in the homogenate to the total cell volume in the homogenate outlined in Supplemental Fig. 1. We observed different concentration levels of HCQ for each cell line, which mostly followed the ranking of MDA-MB$231>$ MDA-MB-468 > T47D > MCF7 (Fig. 1A). For all four $\mathrm{hBC}$ lines, the HCQ total cellular concentrations increased the longer the cell lines were exposed to the drug, up to total cellular concentrations peaking between 0.58 and $2.67 \mathrm{mM}$, which were 58-267 times the extracellular concentration of HCQ, and are consistent with steady-state partition coefficients of HCQ tissue:plasma from previous in vivo studies (McChesney et al., 1967; McChesney, 1983; Wei et al., 1995).

As acidic organelles are suggested to account for a majority of cellular distribution of diprotic lipophilic compounds, we investigated the total lysosomal volume fraction of each cell line using the microscope imaging methods outlined in Supplemental Fig. 2. We observed lysosomal percent volume of total cellular volume ranging from $0.50 \% \pm 0.19 \%$ in MCF7 cells to roughly $3.67 \% \pm 1.09 \%$ in MDA-MB-231 cells (Fig. 1B), which followed roughly the same trend as total cellular uptake of HCQ based on $\mathrm{AUC}_{0-24}$ hour (Fig. 1C). Overall, mean total cell uptake concentrations of HCQ showed a significant

Pearson correlation with lysosomal volume fraction for time points 5, 15, 30, 60, 240, and 1440 minutes (Fig. 1D). The 1minute time point was not significant, likely due to insufficient time for HCQ to diffuse into the lysosomal compartment. Total exposure to HCQ, as calculated by $\mathrm{AUC}_{0-24}$ hour, also had a strong correlation $\left(P=0.032, R^{2}=0.936\right)$ with cellular lysosomal volume fraction (Fig. 1E).

Basal Lysosomal PK Model of HCQ Accounts for Uptake by Adjusting Lysosomal Volume Fraction. To further investigate the in vitro PK of $\mathrm{HCQ}$ in the four $\mathrm{hBC}$ lines, we used a base PK model of lysosomotropic drug uptake (Trapp et al., 2008; Kornhuber et al., 2010) and modified it to be specific to the four cell lines in the study with experimentally determined lysosomal volume fractions. HCQ in vitro PK was modeled as a diffusion-based three-dimensional system in which net flux of HCQ across membranes is driven by the permeability of each ionization state, and ionization state $(0$, $1^{+}, 2^{+}$) is a function of $\mathrm{pH}$ described by the HendersonHasselbalch activity ratio. The model consisted of three compartments representative of in vitro settings: culture media, cytosol, and lysosomes (Fig. 2A). Trafficking between compartments was driven by net flux. The lysosomal compartment had a dynamic $\mathrm{pH}(\Delta \mathrm{pH})$ term that was a function of lysosomal HCQ concentration, as HCQ is suggested to neutralize the acidic lysosomal $\mathrm{pH}$. Model parameters are listed in Supplemental Table 1.

By adjusting the lysosomal volume fractions for each cell line, we simulated the in vitro PK experiment of HCQ administered at $10 \mu \mathrm{M}$ for each cell line out to 1 hour (Fig. 2B). For all cell lines except T47D, the experimentally derived mean lysosomal volume fraction allowed the simulation to capture the mean concentration of $\mathrm{HCQ}$ with a strong fit. The simulation was also

B

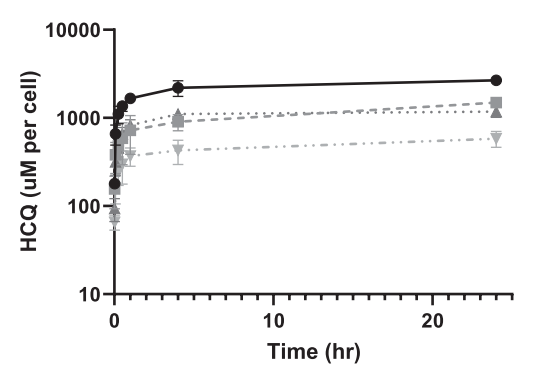

D

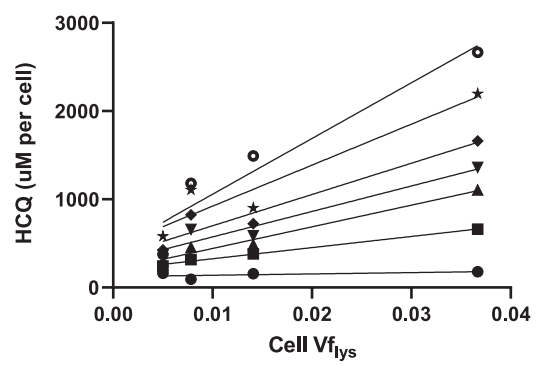

$\rightarrow$ MDA-MB-231

-1. MDA-MB-468

A. T47D

-v. MCF7

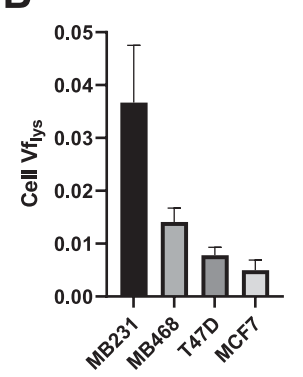

E

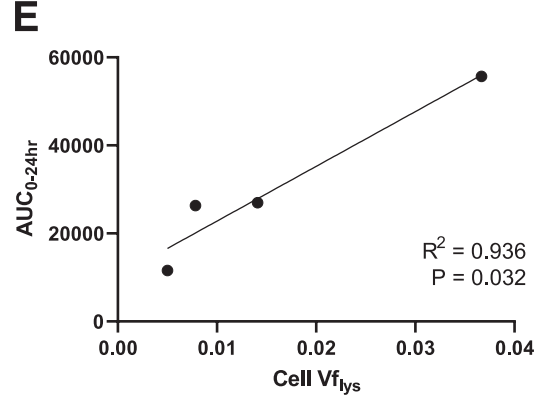

Fig. 1. Whole-cell uptake of HCQ is proportional to basal lysosomal volume fraction. The in vitro whole-cell PK of HCQ was assessed in four hBC cell

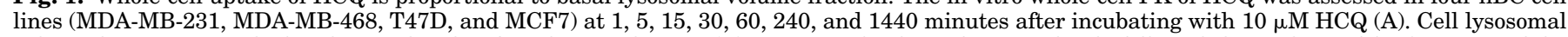

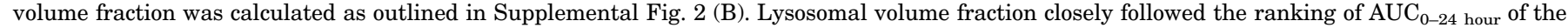

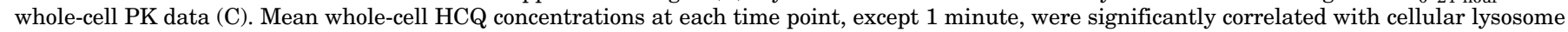

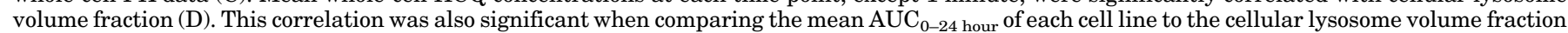
(E). Applicable data are shown as means \pm S.D., and significance is defined as $P<0.05(*)$ and $\mathrm{P}<0.01(* *)$. 
A

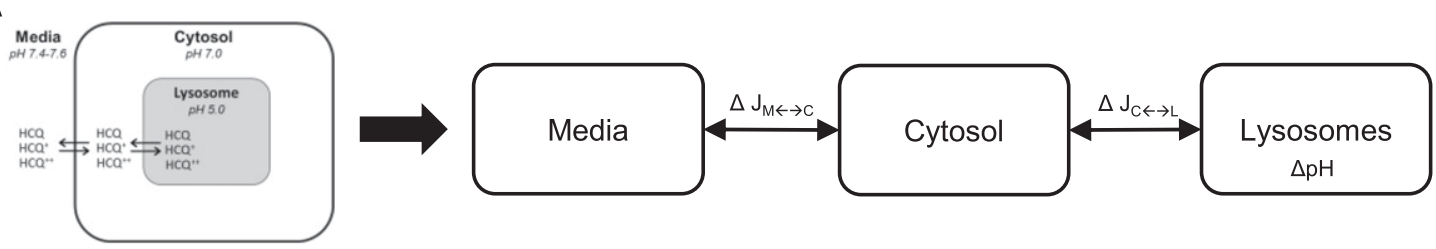

B

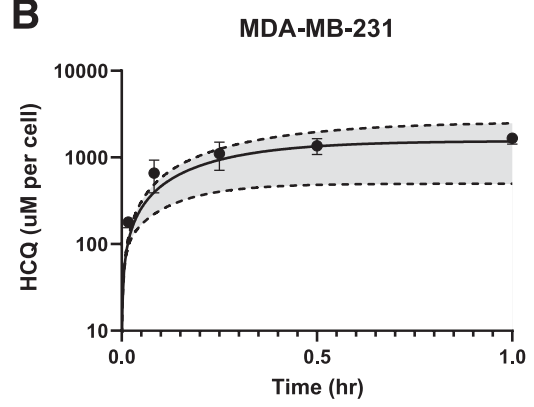

T47D

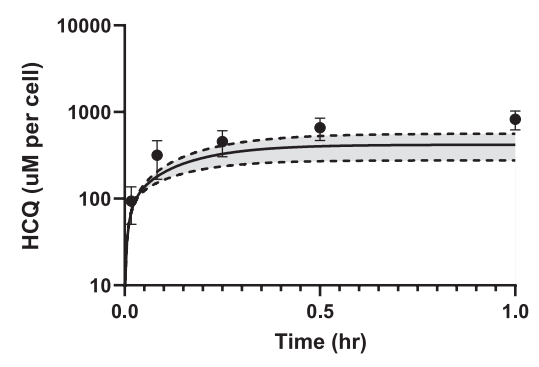

C

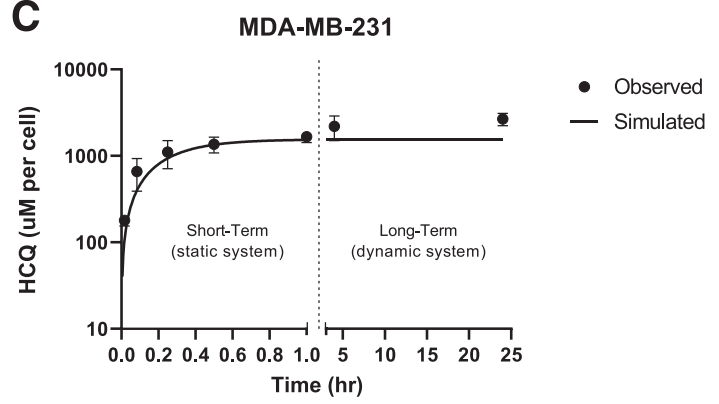

MDA-MB-468

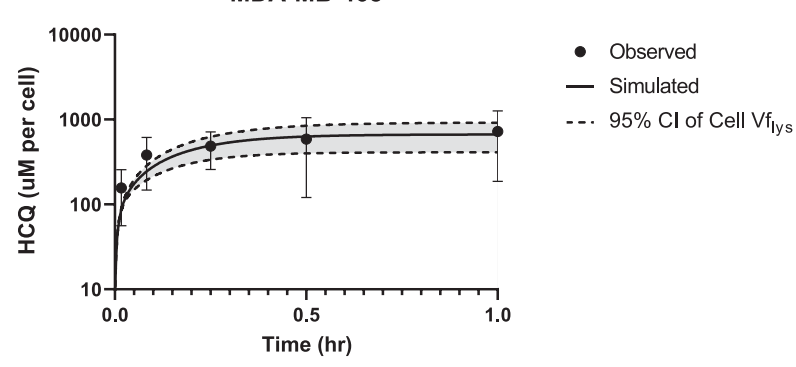

MCF7

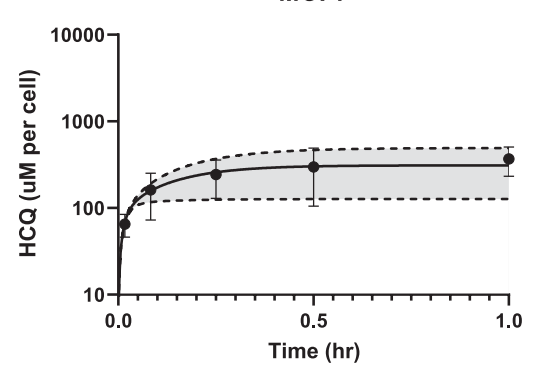

Fig. 2. Basal lysosome PK model of HCQ accounts for uptake by adjusting lysosomal volume fraction. To further investigate the in vitro PK of HCQ in the $4 \mathrm{hBC}$ lines, we used a previously published base model of lysosomotropic drug uptake into cells. The compartmental model is outlined, in which compartments considered are culture media, cytosol, and lysosome, with the lysosome contained within the cytosolic compartment (A). Mathematically, the three compartments are separate; HCQ diffuses freely from media $(\mathrm{M}) \leftrightarrow$ cytosol $(\mathrm{C}) \leftrightarrow$ lysosomes $(\mathrm{L})$, and diffusion is represented by a net flux term $(\Delta J)$ that is the sum of permeability of each HCQ ionization state (neutral,,+++ ). The lysosomal compartment has a dynamic $\mathrm{pH}$ feedback term that was representative of the proposed mechanism of HCQ to increase the $\mathrm{pH}$ of the lysosome based on free drug concentration. The model was used to simulate HCQ uptake in each cell line based on lysosomal volume fractions and other parameters in Table 1. The output of the model simulation for each cell line is shown as the observed mean $\pm 95 \%$ CI bounds in conjunction with the simulated mean $\pm 95 \%$ CI bounds of the cellular lysosomal volume fraction (B). The model only considered time points out to the 1st hour, which were labeled as short-term static equilibrium. In the experimental data, it is observed that concentrations continue to increase after 1 hour, which is characterized as the long-term dynamic system (C), and this is investigated in later figures.

run using the $95 \%$ CI bounds for the experimentally derived lysosomal volume fractions to investigate variability due to this parameter. The simulation suggested that the system reaches equilibrium around 30 minutes; however, the experimental PK data for all four cell lines suggests that HCQ concentrations in the cell continue to increase steadily at 1,4 , and 24 hours (Fig. 2C). Early time points were characterized by chemical kinetic equilibrium, in which the cellular physiologic parameters are static, followed by later time points when the system physiologic parameters are dynamic. The dynamic system is investigated further in later figures. The model fit for each of the cell lines out to 1 hour is characterized by the ratios of simulated:observed AUC (Table 1), as well as measures of predictive performance PE, MAPE\%, and MPE $\%$ (Supplemental Table 2). Using a previously determined range of $0.5-2.0$ as acceptable ratios for AUC of simulated: experimental, the model captured MDA-MB-231, MDA-MB468 , and MCF7 very closely $(0.936,0.995,0.980)$. T47D was 
TABLE 1

Static model metrics (AUC)

\begin{tabular}{lccc}
\hline AUC Summary (0 to $1 \mathrm{~h}$ ) & $\begin{array}{c}\text { Experimental } \\
\text { (Exp) } \\
\text { Mean }\end{array}$ & $\begin{array}{c}\text { Simulated } \\
\text { (Sim) } \\
\text { Mean }\end{array}$ & $\begin{array}{c}\text { Ratio } \\
\text { Mean Sim/Exp }\end{array}$ \\
\hline MDA-MB-231 & 1239 & 1160 & 0.936 \\
MDA-MB-468 & 551 & 549 & 0.995 \\
T47D & 588 & 354 & 0.602 \\
MCF7 & 276 & 270 & 0.980 \\
\hline
\end{tabular}

underpredicted but still fell within the acceptable range (0.602). In comparing the model PE, the model underpredicted early time points (1, 5 minutes) for all cell lines except MCF7. Time points between 15 and 60 minutes were predicted with good accuracy, aside from T47D. MAPE\%, a metric of model accuracy, suggests that the model predicts individual time points in MDA-MB cell lines with about 30\% error, T47D around $60 \%$, and MCF7 about 6.5\%. Overall, the model underpredicts concentrations (MPE\%) by 18.4 and $8.6 \%$ in the MDA-MB cell lines, although this is biased by early time point error, $64 \%$ in $\mathrm{T} 47 \mathrm{D}$, and overpredicts by only $1.3 \%$ in MCF7. Observations from these metrics suggest that T47D cell uptake of HCQ may be better explained by the upper CI bound of its lysosomal volume fraction (1.16\%) rather than its mean $(0.78 \%)$. Model metrics also suggest that early time points (1, 5 minutes) in MDA-MB cell lines were either underpredicted based on uptake kinetics or there was time-based experimental error exacerbated by early time point sampling.

We also investigated the influence of the $\mathrm{pH}$ parameters on total cellular uptake of HCQ in MDA-MB-231 cells. We measured the $\mathrm{pH}$ of incubator-conditioned culture media $\left(5 \% \mathrm{CO}_{2}, 37^{\circ} \mathrm{C}\right)$ used in the previous experiments at 7.6 and made acidic culture media at an incubator-conditioned $\mathrm{pH}$ of 7.0. As stated in the Materials and Methods section, we opted out of using culture media buffers other than sodium bicarbonate, such as HEPES, as it has been suggested to activate the micropthalmia/transcription factor $\mathrm{E}$ (MiT/TFE) pathway, which ultimately results in lysosomal biogenesis (Tol et al., 2018). As the model accurately captured MDA-MB231 cell lines under neutral conditions, they were exposed to 1 or $10 \mu \mathrm{M} \mathrm{HCQ}$ in the conditioned culture media for 1 hour, and the resulting decrease in total cellular uptake of $\mathrm{HCQ}$ associated with decreasing media $\mathrm{pH}$ was quite dramatic. We observed a 6.4-fold decrease in HCQ uptake at the treated concentrations when switching the media $\mathrm{pH}$ from 7.6 to 7.0 such that in most cases the $10 \mu \mathrm{M}$ HCQ uptake at $\mathrm{pH}$ of 7.0 was nearly equivalent to the $1 \mu \mathrm{M}$ HCQ uptake at $\mathrm{pH}$ of 7.6 (Supplemental Fig. 3A). The shape of the simulated kinetics uptake curve was not visibly modified by extracellular $\mathrm{pH}$ out to the 1-hour time point tested (Supplemental Fig. 3B). Interestingly, lysosomotropic drug partition into cells and tissues has been suggested to be significantly reduced by acidic extracellular pH (Wojtkowiak et al., 2011), causing a subsequent resistance of cells to growth inhibition by HCQ. We tested the effect of acidic culture media in blunting HCQ growth inhibition in MDA-MB-231 cells and observed an almost complete reduction in growth inhibition (Supplemental Fig. 3C), similar to the trend described with CQ (Pellegrini et al., 2014). In addition to testing the influence of extracellular $\mathrm{pH}$ directly, we also investigated the contribution of lysosome and cytosol $\mathrm{pH}$ gradient indirectly by treating cells with MN prior to HCQ to eliminate the gradient. In MDA-MB231 cells, pretreatment with $25 \mu \mathrm{M}$ MN for 30 minutes prior to HCQ caused a reduction in total cellular uptake of HCQ by $93.2 \%$ at 1 hour. We simulated this experiment using the model by changing the lysosomal $\mathrm{pH}$ to 7.0 and comparing with the model with lysosomal $\mathrm{pH}$ of 5.0 (Supplemental Fig. $3 \mathrm{D})$. Overall, the model captured the decrease in total cellular uptake well, with simulated $\mathrm{MN}(+): \mathrm{MN}(-)$ concentration ratio of 0.078 versus observed $\mathrm{MN}(+): \mathrm{MN}(-)$ concentration ratio of 0.068 .

HCQ Increases the Size of the Lysosomal Compartment. Although the model captures early time point in vitro PK ( $<1$ hour) of HCQ, as well as variabilities in influential model parameters along the $\mathrm{pH}$ gradient, it fails to capture later time points, as the simulation suggests the system reaches equilibrium when the observed concentrations actually continue to gradually increase out to 24 hours (Fig. 2C). Recent reports suggest that many weakly basic lipophilic compounds, including CQ, activate lysosomal biogenesis by activating TFEB (Lu et al., 2017; Zhitomirsky et al., 2018; Zhao et al., 2020), which ultimately increases the lysosomal volume fraction of treated cells and should allow them to sequester even more drug than their original baseline (Ruzickova et al., 2019). CQ has been observed to activate TFEB and increase lysosomal content of the cell, so we investigated the capability of HCQ to do the same. Treatment of all four hBCs with either HCQ for 24 hours or TFEB activator Torin1 for 16 hours caused a significant increase in TFEB activity in the nucleus (Fig. 3A), suggesting it is released from the lysosome and translocated to the nucleus after HCQ treatment. In addition, gene microarray data on all four cell lines treated with HCQ or vehicle for 24 hours showed a significant enrichment in expression of the TFEB-associated direct targets in lysosomal function (Fig. 3B) as characterized by the Ballabio group (Palmieri et al., 2011). Treatment of all four cell lines with HCQ or Torin1 caused an increase in the relative volume of the lysosome compartment. MCF7 cells had the most visually distinct increase in lysosomal burden with both drugs (Fig. 3D). Quantitation of the increase in lysosomepositive area per nuclei suggests a relative increase of 1.59- to 14.93-fold (average 5.90-fold) in the cell lines with 24-hour treatment with HCQ and a 2.25- to 10.7-fold (average 5.55fold) increase with Torin 1 (Fig. 3C). This increase in lysosomal burden by HCQ may have some contribution independent of TFEB as well, as CQ has been shown to induce swelling in lysosomes at higher doses, which is visually apparent in images comparing Torin 1 increase in lysosomal burden versus HCQ increase in lysosomal burden in MCF7 (Fig. 3D) and the other three cell lines (Supplemental Fig. 4). To further investigate lysosomal swelling by HCQ, we transfected MDA-MB-468, T47D, and MCF7 cells with a GFP baculovirus for lysosomal associated membrane protein 1, as it more clearly defines the membranes of individual lysosomes, and we imaged cells after treatment with $10 \mu \mathrm{M}$ HCQ for 24 hours. MDA-MB-231 cells would not express the virus and were omitted from this experiment. In the three cell lines, we observed a dramatic increase in size of some lysosomes after HCQ exposure (Supplemental Fig. 5).

Dynamic Lysosomal Volume Accounts for Simulation Error. The original model with a static lysosomal volume compartment was able to capture total cell uptake of HCQ out to 1 hour but significantly underpredicted 4 - and 24-hour time 
A

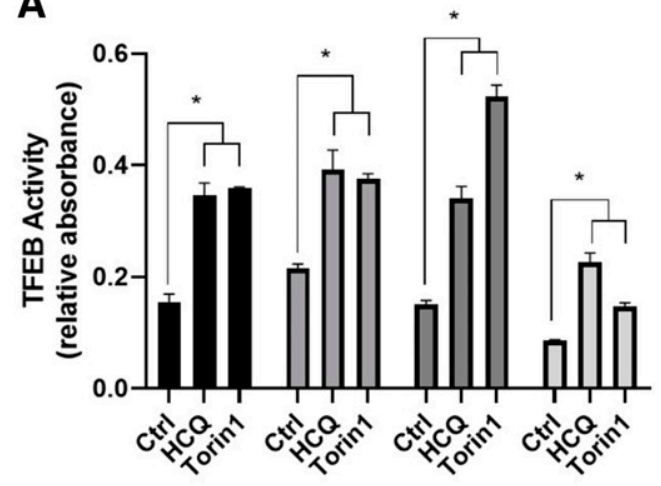

C

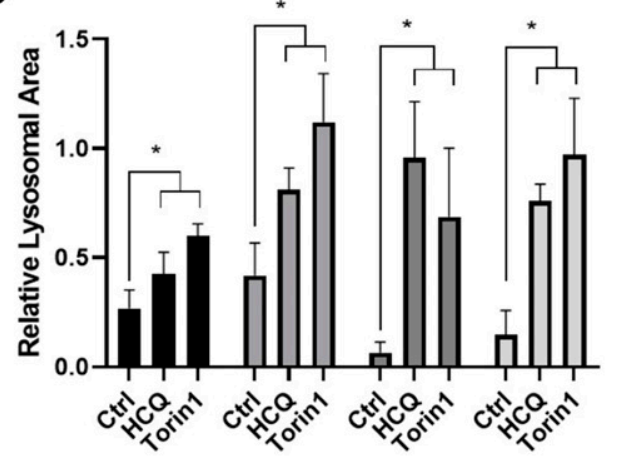

B

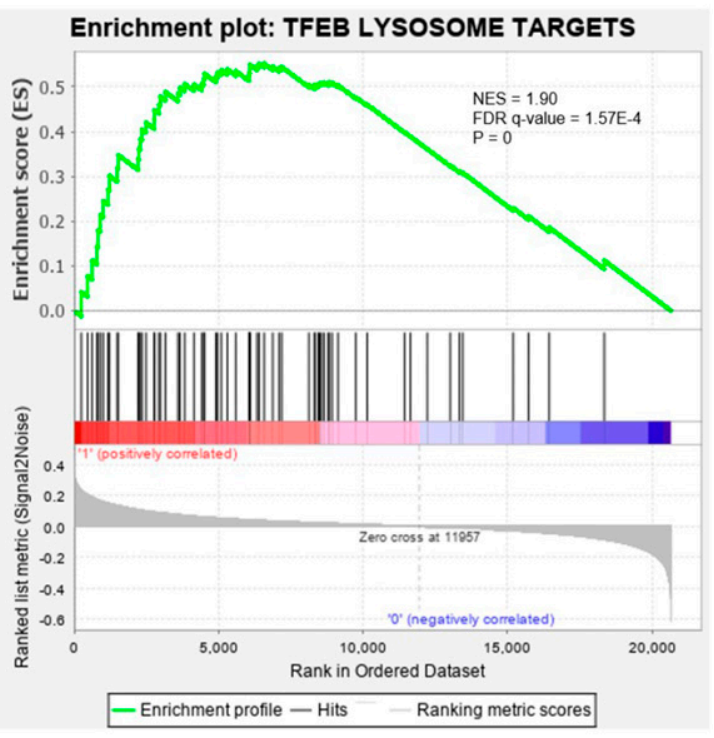

D Nuclei Lysosomes Merge

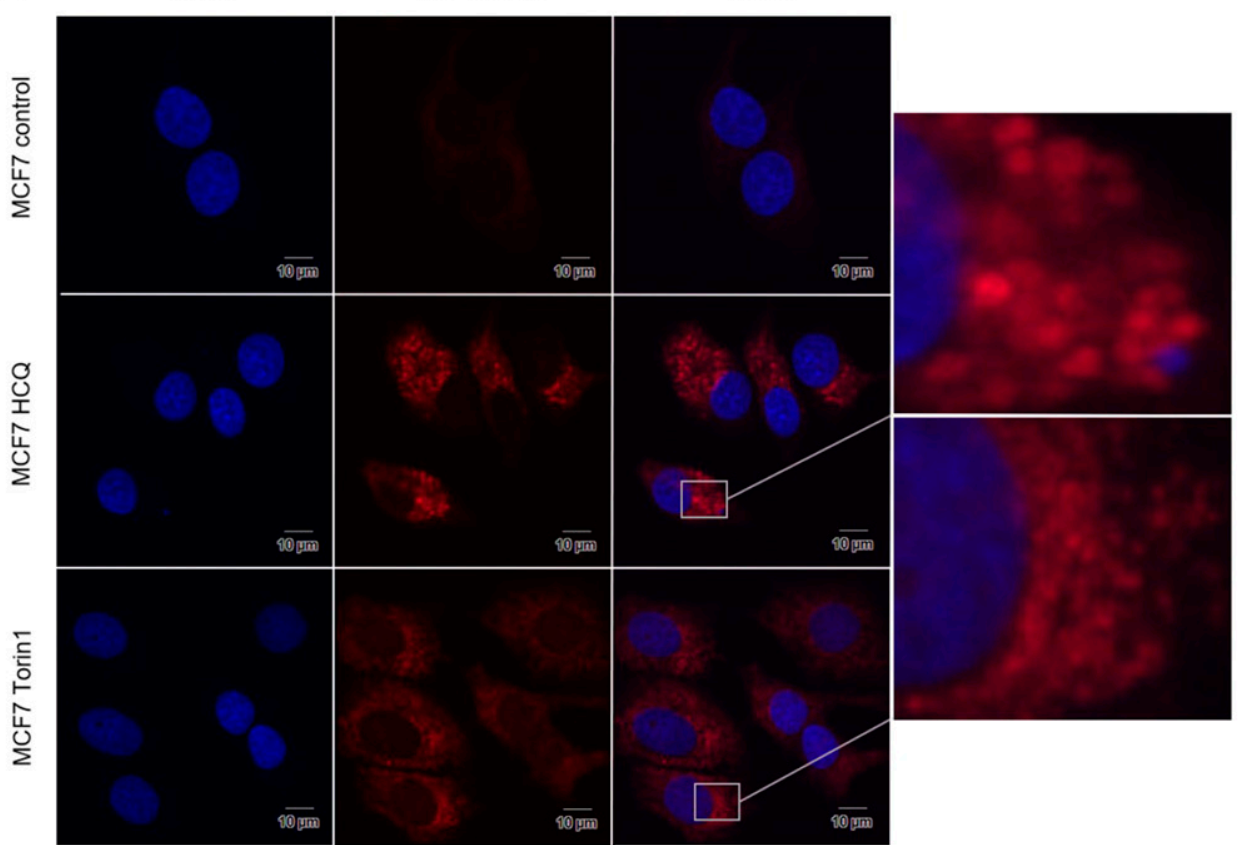

- MDA-MB-231

口 MDA-MB-468

口 T47D

ㅁ MCF7

Fig. 3. HCQ increases the size of the lysosomal compartment. We investigated the ability of HCQ to change the size of the lysosomal compartment. TFEB activity in the nucleus was significantly increased in all cell lines treated with $10 \mu \mathrm{M}$ HCQ for 24 hours and with 250 nM Torin 1 , a molecular TFEB-activating agent, for 16 hours (A). GSEA of all four cell lines treated with $20 \mu \mathrm{M}$ HCQ for 24 hours also caused a significant enrichment of TFEB lysosome targets, as determined by the normalized enrichment score (NES) and false discovery rate (FDR) (B). Fluorescence microscopy imaging of all four cell lines with ETP showed a significant increase in lysosome accumulation within all cell lines treated with $10 \mu \mathrm{M}$ HCQ for 24 hours or $250 \mathrm{nM}$ Torin 1 for 16 hours (C). Representative images of MCF7 cells imaged with ETP under the treatment conditions are shown in in (D). Zooming in on MCF7 cells treated with HCQ shows a visual increase in lysosomal size in comparison with the Torin 1 treatment. Significance is defined as $P<0.05(*)$. To prepare figures for publication, the raw image threshold was adjusted to the same upper and lower bounds across the entire image for all images shown. 
points. To account for this error, we incorporated lysosomal biogenesis into the model by adding a dynamically growing lysosomal volume fraction represented by the proposed mechanism outlined in Fig. 4. The growing compartment is represented as a linear increase in lysosomal volume fraction as a function of time: $\mathrm{Vf}_{\mathrm{lys}}(\mathrm{t})=\mathrm{Vf}_{\mathrm{lys}_{\mathrm{y}} 0} \bullet(1+\mathrm{x} \bullet \mathrm{t})$. We found that a 1.5- to 3-fold total increase in lysosomal volume fraction by 24 hours in the dynamic lysosomal model accounted for the underprediction of 4- and 24-hour time points predicted by the static lysosomal model (Fig. 5A). The simulated 1.5- to 3-fold increase in lysosomal volume fraction over 24 hours of HCQ exposure was within the $95 \%$ CI of the experimentally observed increase due to HCQ in the four cell lines shown in Fig. 3C, except for T47D. As such, for T47D, we tested an increase of 2.5fold in lysosomal volume starting from the upper bound of the 95\% CI $(1.155 \%)$, which resulted in a better model fit to 24 hours. Incorporation of this time-based linear increase did not significantly affect time points before 1 hour (Fig. 5B).

The model fit, as characterized by the ratios of simulated: observed $\mathrm{AUC}_{0-24}$ hour, is shown in Table 2, and measures of predictive performance PE, MAPE\%, and MPE\% are shown in Supplemental Table 3. Overall, the model captured the early time points of the cell lines with good performance, as the AUC and ratios were close to 1 . PE followed the same trend to the static model for time points 0-1 hour, with 4- to 24-hour time points being slightly underpredicted for all cell lines except MCF7 and T47D at 24 hours. MAPE\% suggests the model fits overall time points for all cell lines between $7.53 \%$ and $45.21 \%$, and MPE\% suggests a slight underprediction bias for the model. Overall, this experimental data and model simulation suggest that HCQ creates a positive-feedback loop within the cell lysosomal compartment, causing it to increase its own distribution within the cell over time.

Torin1 Increases Size of Lysosomal Compartment, Which Increases Cell Uptake of HCQ. Although HCQ appears to be able to modify its own distribution, we investigated the capability of direct modification of the cell lysosomal volume fraction to increase HCQ uptake capacity. In Fig. 4D, we observed that Torin 1 increased the relative lysosomal burden by 2.25- to 10.7 -fold. In this experiment, we repeated the HCQ cell uptake experiments out to 24 hours but first pretreated cells with Torin1(+) or (-) for 16 hours prior to administering HCQ. Pretreatment with Torin1 resulted in a mean increase in cellular uptake of HCQ at all time points in all cell lines of 1.4- to 1.6 -fold (Fig. 6A). To test this in the context of the in vitro PK model, we changed the cell line base lysosomal volume fraction at time 0 based on the mean fold increase of lysosomal fraction by Torin 1 from Fig. 3 and simulated the dynamic lysosome PK model (Fig. 6A). The dynamic lysosome PK simulation suggested that this change in initial lysosome starting fraction accounts for the increased uptake of HCQ in the cells. For the new data set, the MDAMB-468 T1(-) uptake data were simulated against the lower CI basal $\mathrm{Vf}_{\mathrm{lys}}(0.835 \%)$ and the $\mathrm{T} 47 \mathrm{D}$ against the mean starting $\mathrm{Vf}_{\mathrm{lys}}(0.783 \%)$ from Fig. 1B. In MDA-MB-231 and 468 cells, the mean increase of lysosomes by Torin 1 accounted for the increased cell uptake of HCQ in the simulation. In T47D and MCF7, the experimental mean increase in lysosomes by Torin 1 from Fig. $3 \mathrm{C}$ was very high, so the ratio from the lowest replicate was used to show the increase the model would account for. Comparison of observed versus simulated $\mathrm{AUC}_{0-24}$ hour are shown in Table 3 . All simulation:experimental ratios except the T47D and MCF7 mean T1 were within the range of $0.5-2.0$; however, T47D and MCF7 simulations using the lowest $\mathrm{T} 1 \mathrm{Ctrl}$ lysosome ratio from a replicate did fall within this range. Model performance metrics are shown in Supplemental Table 4. The model for each cell line followed similar PE trends to the earlier versions of the models. MAPE $\%$ for all cell lines suggests that the model fits overall time points between $15.48 \%$ and $61.69 \%$, and MPE\% suggests an even spread of over- and underprediction of the model across the cell lines. Overall, the cell PK model of HCQ supports that steadily increasing long-term whole-cell concentrations, as well as the increase in concentration caused by Torin 1 pretreatment, are linked directly with the increase in size of the lysosomal compartment observed experimentally.

In addition, we ran the same experiment in MDA-MB-231 cells, except cells were pretreated with $\mathrm{T} 1, \mathrm{~T} 1+\mathrm{MN}$, or $\mathrm{MN}$ alone prior to treatment with HCQ for 1 hour. We observed that the ratio of T1:Ctrl was not statistically significant from the ratio of $\mathrm{MN}-\mathrm{T} 1: \mathrm{MN}-\mathrm{Ctrl}$ (1.6), suggesting that this increase in cell uptake of HCQ is due to only the increase in lysosomal volume fraction induced by T1 (Fig. 6C). In MDAMB-231, model simulation of the T1(+) versus $\mathrm{T} 1(-)$ scenarios $\begin{array}{ll}\text { 1. } \mathrm{HCQ} \text { administered } & \text { 2. } \mathrm{HCQ} \text { accumulates in }\end{array}$ lysosomes
3. $\mathrm{HCQ}$ causes a) lysosomal swelling, and b) activation of TFEB
4. Lysosomes accumulate within the cell, increasing the total magnitude of $\mathrm{HCQ}$ distribution in the cell

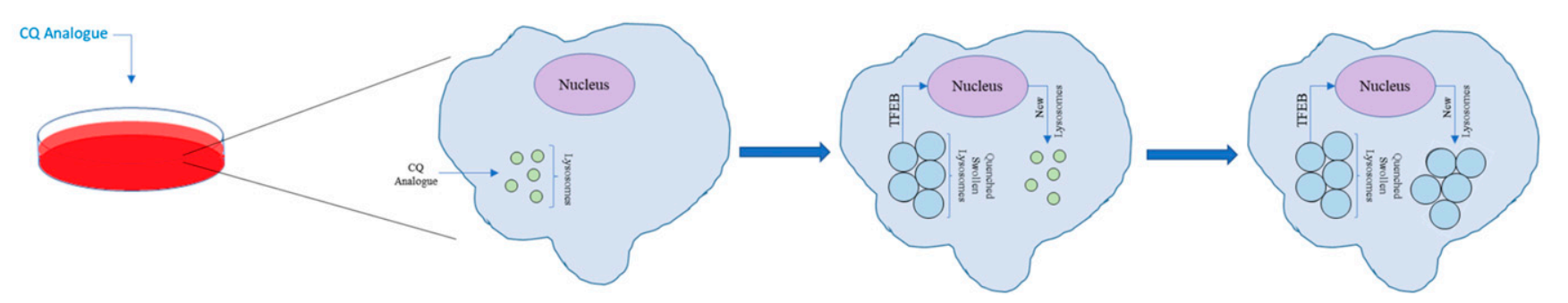

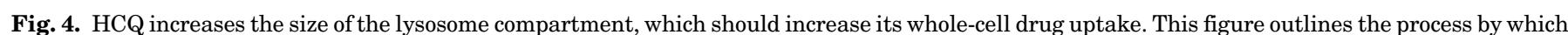

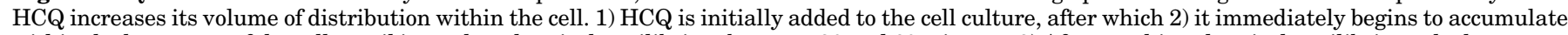

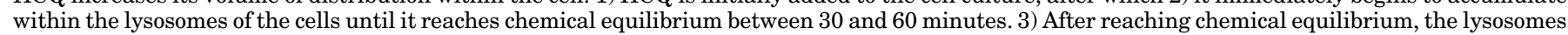

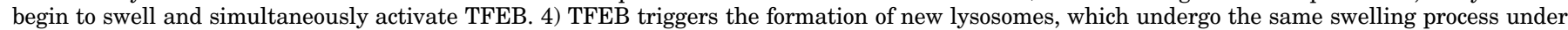

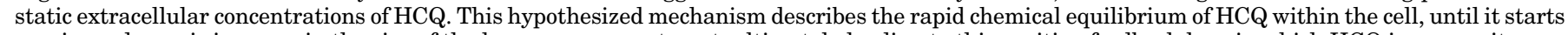

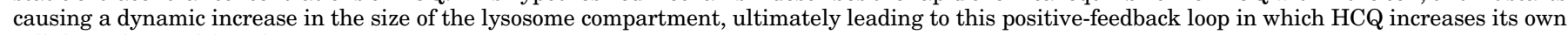
cellular volume of distribution. 
A

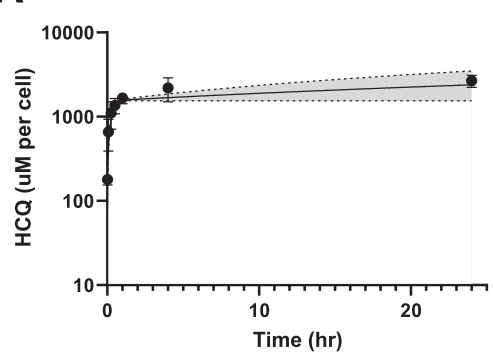

- Observed

— Simulation $\left(1.60^{*} \mathrm{Vf}_{\text {lys }}\right)$

No Growth $\left(1.00^{\star} V f_{\mid y s}\right)$

Upper $\left(2.40^{\star} \mathrm{Vf}_{\text {lys }}\right)$
T47D

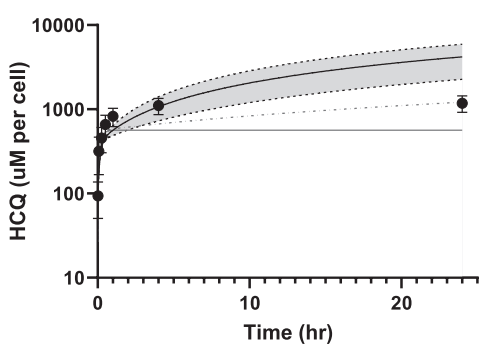

MDA-MB-468

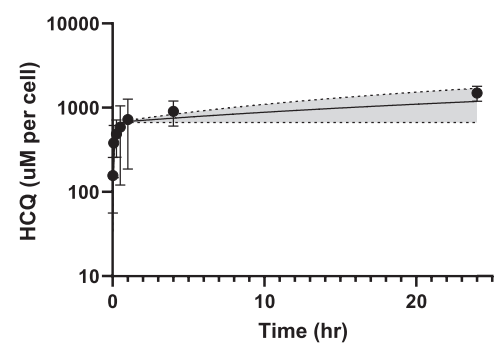

- Observed

— Simulated $\left(1.96^{\star} \mathrm{Vf}_{\mathrm{lys}}\right)$ No Growth $\left(1.00^{*} \mathrm{Vf}_{\text {lys }}\right)$ Upper $\left(2.94^{*} \mathrm{Vf}_{\text {lys }}\right)$

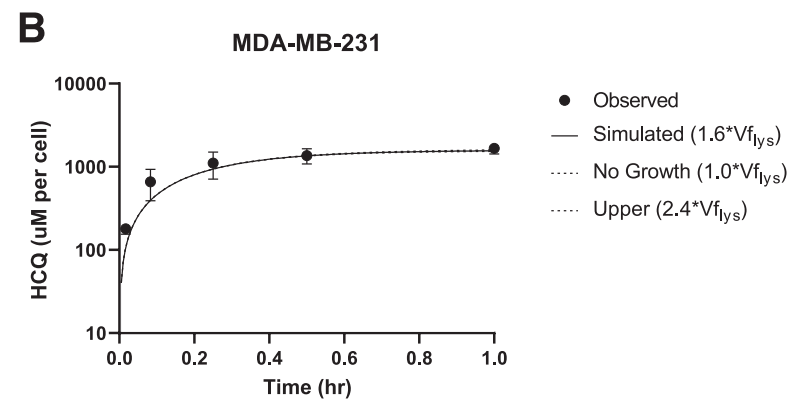

- Observed

- Simulated $\left(14.93^{*} \mathrm{Vf}_{\mathrm{lys}}\right)$

- No Growth $\left(1.00^{*} \mathrm{Vf}_{\text {lys }}\right)$ Realistic $\left(2.5^{*} \mathrm{Vf}_{\mid \mathrm{lys}}\right)$

.... Lower $\left(7.47^{\star} \mathrm{Vf}_{\text {|ys }}\right)$ Upper $\left(22.40^{*} \mathrm{Vf}_{\text {lys }}\right)$

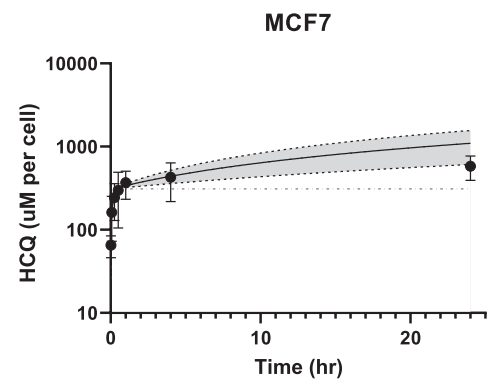

- Observed

— Simulated $\left(5.14^{*} \mathrm{Vf}_{\mid y s}\right)$

.. No Growth $\left(1.00^{*} V f_{\mid y s}\right)$

..... Lower $\left(2.57^{\star} \mathrm{Vf}_{\text {lys }}\right)$

..... Upper $\left(7.70^{*} \mathrm{Vf}_{\mathrm{lys}}\right)$

Fig. 5. Dynamic lysosome volume accounts for simulation error in long-term HCQ uptake. To investigate HCQ whole-cell uptake past 1 hour, we incorporated a growing lysosomal component into the model for all cell lines. Lysosomal growth is represented by a linear increase as a function of time, out to a maximum lysosomal volume of the fold increase observed with HCQ treatment of each cell line in Fig. 3C. The dynamic system model is shown (A) for MDA-MB-231, MDA-MB-468, T47D, and MCF7, respectively. MDA-MB-231 and MDA-MB-468 simulated uptake is shown as the mean increase in experimental lysosomal volume after HCQ treatment, the upper value of the lysosomal volume, and with no growth incorporated. T47D is shown as the mean, upper, and lower increase in lysosomal volume as well as the value we would expect based on the PK data (realistic) and no growth. MCF7 is shown as the mean, upper, lower, and no growth. It should be noted that the time-based increase in lysosomal volume in the dynamic model does not affect earlier time points and thus would not affect model fit vs. the static model out to 1 hour (B).

represented an increase in total cellular uptake of $\mathrm{HCQ}$ but also showed that the lysosomal concentrations in both conditions was roughly the same (Fig. 6D). This suggests that even though the quantifiable concentration of HCQ from a PK study (whole-cell or tissue homogenate) may change, the concentration in the lysosome may be the same. This observation could have implications for PK:PD correlations, as the lysosome is the target site of HCQ for autophagy inhibition.

TABLE 2

Dynamic model metrics (AUC)

\begin{tabular}{lccc}
\hline $\begin{array}{l}\text { AUC Summary (0-24 h) } \\
\text { Cell Line }\end{array}$ & $\begin{array}{c}\text { Experimental (Exp) } \\
\text { Mean }\end{array}$ & $\begin{array}{c}\text { Simulated (Sim) } \\
\text { Mean }\end{array}$ & Mean Sim/Exp \\
\hline MDA-MB-231 & 55,660 & 46,753 & 0.84 \\
MDA-MB-468 & 26,922 & 22,085 & 0.82 \\
T47D & 26,340 & 56,178 & 2.13 \\
T47D (realistic) & & 21,198 & 0.80 \\
MCF7 & 11,553 & 16,823 & 1.46 \\
MCF7 (lower) & & 10,975 & 0.95 \\
\hline
\end{tabular}

\section{Discussion}

Lysosomes have been observed as a drug sink for many lysosomotropic agents, including many prescribed drugs, such as doxorubicin, vinblastine, and imipramine, and a variety of TKIs, such as gefitinib (Skoupa et al., 2020), imatinib (Burger et al., 2015), and sunitinib (Ruzickova et al., 2019). In a recent study by Ruzickova et al., the lysosomotropic agent sunitinib was shown to be capable of increasing overall lysosomal sequestration of the TKIs tested, although this effect was insufficient in reducing sensitivity to the TKIs by the hypothesized mechanism of sequestering them away from their mechanistic target sites as the concentrations tested in culture maintained a static extracellular concentration. The in vitro PK model of HCQ supports this finding and would suggest the same observation: the direct increase of lysosomal volume fraction by pretreating with Torin1 (Fig. 6C) barely increases HCQ concentration in the lysosome even though the whole-cell concentration is higher. This observation sheds light onto one commonly suggested hypothesis for 
A

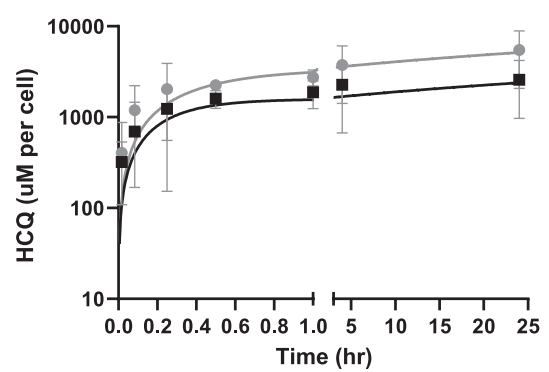

T47D

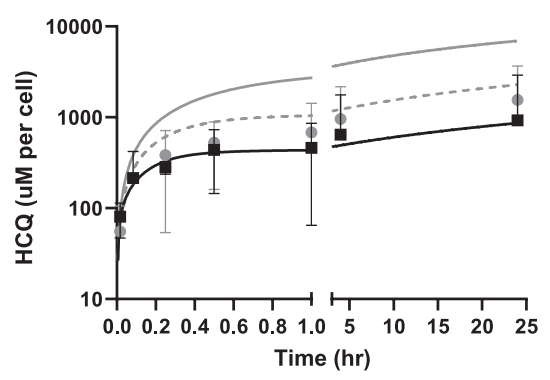

B

MDA-MB-231

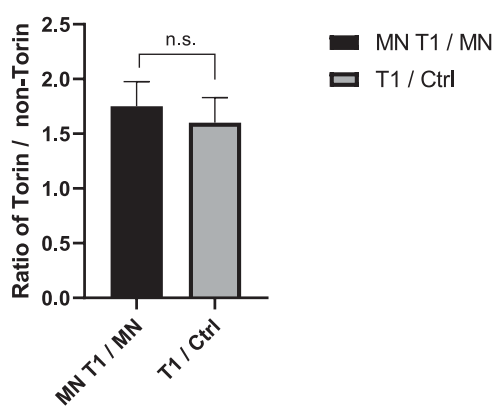

MDA-MB-468

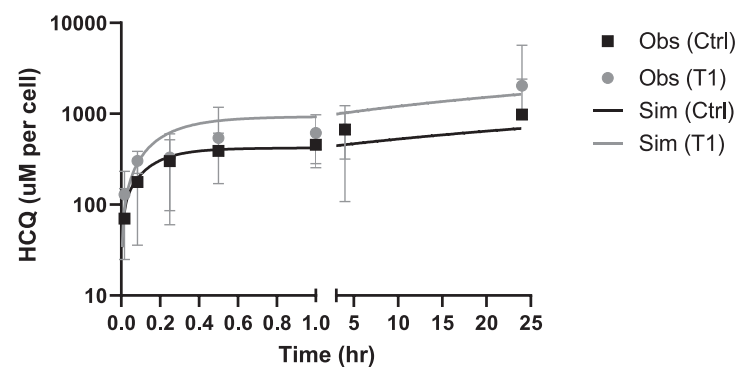

MCF7

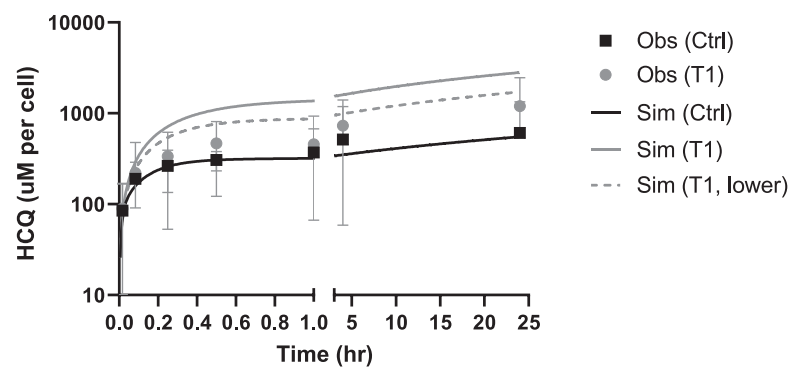

C MDA-MB-231

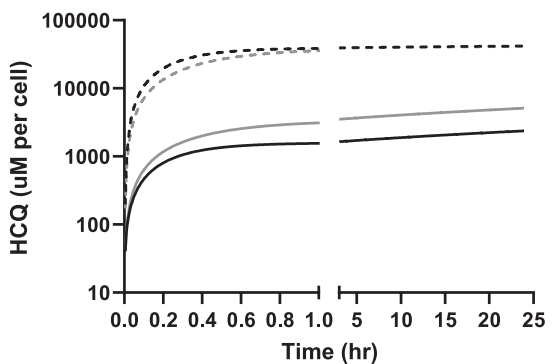

- Ctrl (whole cell)

... Ctrl (lysosome)

- T1 (whole cell)

-.. T1 (lysosome)

Fig. 6. Torin 1 increases the size of the lysosomal compartment, increasing whole-cell uptake of HCQ. Cell lines were pretreated with T1 using the same concentration and time as in Fig. 3. After Torin1 pretreatment, the HCQ PK studies from 1 minute to 24 hours were repeated in Torin1(+) vs. Torin1(-) cells (Obs). Torin1 pretreatment resulted in an average increase of HCQ whole-cell concentrations at all time points by an average of 1.4- to 1.6-fold. The mathematical PK model of HCQ (Sim) was tested at basal lysosomal volume fractions vs. Torin1-modified starting lysosomal volume fractions by multiplying the basal lysosomal volume fraction in each cell line by the mean increase by Torin1 treatment from Fig. 4D (A). MDA-MB-231 [(A), top-left] fit the data well using mean values of Torin1. MDA-MB-468 used the starting lysosomal volume fraction value of the lower $95 \%$ CI (0.753\%) and mean value of Torin 1 increase [(A), top-right]. T47D used the mean starting $\mathrm{Vf}_{\mathrm{lys}}(0.783 \%)$ and was tested against the mean Torin 1 increase in lysosomes $(10.5 \times)$ as well as the lowest observed ratio within the replicates $(3 \times)$ [(A), bottom-left]. MCF7 was tested against the mean lysosomal increase by Torin1 and the lowest observed ratio within the replicates $(3.8 \times)$ [(A), bottom-right]. To test whether the increase in uptake by Torin1 was only due to the increase in lysosomes, MDA-MB-231 HCQ uptake was tested at 1 hour with no pretreatment, Torin1 pretreatment, monensin pretreatment, or Torin1 and monensin pretreatment. No significant change in the ratio between T1:Ctrl and MN + T1:MN HCQ uptake was observed (B) with a two-tailed unpaired $t$ test $(P=0.573)$. Comparing the simulated lysosomal concentrations vs. the whole-cell uptake concentrations in MDA-MB-231 cells with T1( + ) or (-) shows a minimal difference in lysosomal concentration in both scenarios (C).

HCQ growth-inhibition synergism with other lysosomotropic agents in cell culture, which is that HCQ displaces drugs from the lysosome, reducing overall sequestration and increasing concentration at target sites elsewhere within the cell. This work, in conjunction with other recent findings, would suggest that under static extracellular concentrations, this hypothesized mechanism is unlikely, as an overall reduced cellular uptake of lysosomotropic drugs may be due to displacement by HCQ but would not result in a subsequent increase in concentration at the drug's mechanistic target. This does not eliminate this hypothesized mechanism in vivo, though, as extracellular concentrations are not constant.
Many drugs have been shown to activate TFEB in vitro in recent publications (Lu et al., 2017; Zhitomirsky et al., 2018; Zhao et al., 2020), most of which are weakly basic lipophilic agents that would be physicochemically characterized as lysosomotropic. If lysosomotropism is a major factor in the $\mathrm{PK}$ of these drugs, as it is for HCQ, then the distribution of these drugs might be altered by their effect on the lysosome as we have observed for HCQ. Our findings emphasize care in cell-level PK studies of drugs with weakly basic lipophilic structures, especially when attempting to correlate concentration with response. This is especially important when using multiple lysosomotropic agents in combination. 
TABLE 3

Torin1 dynamic model metrics (AUC)

\begin{tabular}{lrrc}
\hline $\begin{array}{l}\text { AUC Summary (0-24 h) } \\
\text { Cell Line }\end{array}$ & $\begin{array}{c}\text { Experimental (Exp) } \\
\text { Mean }\end{array}$ & $\begin{array}{c}\text { Simulated (Sim) } \\
\text { Mean }\end{array}$ & Mean Sim/Exp \\
\hline MDA-MB-231 (Ctrl) & 56,214 & 46,753 & 0.83 \\
MDA-MB-231 (T1) & 104,084 & 99,813 & 0.96 \\
MDA-MB-468 (Ctrl) & 18,490 & 13,141 & 0.71 \\
MDA-MB-468 (T1) & 29,517 & 30,276 & 1.03 \\
T47D (Ctrl) & 17,692 & 15,304 & 0.87 \\
T47D (T1) & 28,044 & 119,838 & 4.27 \\
T47D (T1, lower) & & 39,299 & 1.40 \\
MCF7 (Ctrl) & 12,849 & 10,284 & 0.80 \\
MCF7 (T1) & 21,471 & 49,679 & 2.31 \\
MCF7 (T1, lower) & & 30,585 & 1.42 \\
\hline
\end{tabular}

Regardless, the possibility of occurrence of this lysosomealtered distribution is supported by in vitro studies in other lysosomotropic agents, whether it be through the activation of lysosomal biogenesis or through lysosomal fusion that is independent of biogenesis (Skoupa et al., 2020), but with limited data to suggest it may occur in vivo. HCQ is somewhat unique in this context because of its long half-life combined with often prolonged dosing regimens, which means that tissue will be exposed to relatively high concentrations of the drug for an extended duration. Extended duration of exposure to tissues might exacerbate the lysosomotropic effects due to HCQ that may not necessarily be prevalent with other drugs that are dosed more short term. We are unaware of any longterm preclinical PK studies involving HCQ that investigate multiple time points aside from the work by McChesney (1983). This study looked at a variety of different tissues in rats that were treated with HCQ 6 days per week for months, with time points at $1,2,3$, and 7 months. The key observation from this study is that HCQ concentrations continually increase up to 3 months, at which time dosing is discontinued in the first study. In the 7-month study, HCQ only starts to reach tissue steady state between 3 and 7 months in lung, eye, and muscle but continues to gradually increase in concentration for other tissue. This is particularly interesting because the plasma half-life of HCQ in rats has been observed at around 10.6 hours (Moore et al., 2011), suggesting a disconnect between plasma and tissue steady-state values. The study by Wei et al. (1995) also investigated HCQ dosing in rats and sampled multiple time points of blood over the course of 10 weeks, but it only sampled tissue at the 10-week mark. Interestingly, of the three dosing regimens applied to both male and female rats ( 6 conditions total), only the regimen with $8 \mathrm{mg} / \mathrm{kg}$ in male rats appeared to increase in blood concentration after the first time point at 3 weeks, but it remained steady after that. For the rest of the dosing regimens, HCQ appeared to remain constant, which would suggest that steadily increasing tissue concentrations of HCQ would be undetected by the traditional PK sampling of blood in clinical studies. With limited in vivo data available, we would speculate that lysosomal biogenesis plays a role in longterm tissue uptake, although this effect would likely be less pronounced because of overall lower HCQ concentrations.

This work has implications in HCQ PK, both in vitro and in vivo, particularly in the context of the cancer clinical trial in solid tumors by Barnard et al. (2014), in which there was no correlation between HCQ concentrations in the tumor and plasma. Further data are needed to investigate the concentration/time relationship of this effect and possible maximum values of lysosome volume fractions that are achievable in vitro. For this study, we modeled the lysosomal compartment as increasing linearly with time, although it is more likely that this effect follows a hyperbolic trend-increasing rapidly and leveling off to eventually reach a pseudomaximum. It is also likely that this effect is due to overall exposure to HCQ (AUC) rather than time or concentration alone, but to investigate that, a significant number of time points and concentrations are necessary in conjunction with subsequent $\mathrm{PK}$ analysis to maintain a connection between the lysosomal biogenesis mechanism and PK outcome. In addition, a comprehensive PK/PD study connecting increasing tissue concentrations over the long term with an increase in lysosomal volume of tissues is warranted to truly confirm whether this phenomenon is important to consider in vivo.

\section{Authorship Contributions}

Participated in research design: Collins, Gustafson.

Conducted experiments: Collins, Witta.

Contributed new reagents or analytic tools: Collins, Coy, Pang.

Performed data analysis: Collins.

Wrote or contributed to the writing of the manuscript: Collins, Gustafson.

\section{References}

Abeywickrama CS, Wijesinghe KJ, Stahelin RV, and Pang Y (2019) Lysosome imaging in cancer cells by pyrene-benzothiazolium dyes: an alternative imaging approach for LAMP-1 expression based visualization methods to avoid background interference. Bioorg Chem 91:103144.

Barnard RA, Wittenburg LA, Amaravadi RK, Gustafson DL, Thorburn A, and Thamm DH (2014) Phase I clinical trial and pharmacodynamic evaluation of combination hydroxychloroquine and doxorubicin treatment in pet dogs treated for spontaneously occurring lymphoma. Autophagy 10:1415-1425.

Belhoussine R, Morjani H, Sharonov S, Ploton D, and Manfait M (1999) Characterization of intracellular $\mathrm{pH}$ gradients in human multidrug-resistant tumor cells by means of scanning microspectrofluorometry and dual-emission-ratio probes. Int $J$ Cancer 81:81-89.

Burger H, den Dekker AT, Segeletz S, Boersma AW, de Bruijn P, Debiec-Rychter M, Taguchi T, Sleijfer S, Sparreboom A, Mathijssen RH, et al. (2015) Lysosomal sequestration determines intracellular imatinib levels. Mol Pharmacol 88:477-487. Duvvuri M and Krise JP (2005) A novel assay reveals that weakly basic model compounds concentrate in lysosomes to an extent greater than $\mathrm{pH}$-partitioning theory would predict. Mol Pharm 2:440-448.

Gallagher LE, Radhi OA, Abdullah MO, McCluskey AG, Boyd M, and Chan EYW (2017) Lysosomotropism depends on glucose: a chloroquine resistance mechanism Cell Death Dis 8:e3014.

Grinde B (1983) Effect of carboxylic ionophores on lysosomal protein degradation in rat hepatocytes. Exp Cell Res 149:27-35.

Gustafsson LL, Ebling WF, Osaki E, Harapat S, Stanski DR, and Shafer SL (1992) Plasma concentration clamping in the rat using a computer-controlled infusion pump. Pharm Res 9:800-807.

Ishizaki J, Yokogawa K, Ichimura F, and Ohkuma S (2000) Uptake of imipramine in rat liver lysosomes in vitro and its inhibition by basic drugs.J Pharmacol Exp Ther 294:1088-1098.

King MA, Ganley IG, and Flemington V (2016) Inhibition of cholesterol metabolism underlies synergy between mTOR pathway inhibition and chloroquine in bladder cancer cells. Oncogene 35:4518-4528.

Kornhuber J, Henkel AW, Groemer TW, Städtler S, Welzel O, Tripal P, Rotter A, Bleich S, and Trapp S (2010) Lipophilic cationic drugs increase the permeability of lysosomal membranes in a cell culture system. J Cell Physiol 224:152-164.

Lu S, Sung T, Lin N, Abraham RT, and Jessen BA (2017) Lysosomal adaptation: how cells respond to lysosomotropic compounds. PLoS One 12:e0173771.

Maycotte P, Gearheart CM, Barnard R, Aryal S, Mulcahy Levy JM, Fosmire SP Hansen RJ, Morgan MJ, Porter CC, Gustafson DL, et al. (2014) STAT3-mediated autophagy dependence identifies subtypes of breast cancer where autophagy inhibition can be efficacious. Cancer Res 74:2579-2590.

McChesney EW (1983) Animal toxicity and pharmacokinetics of hydroxychloroquine sulfate. Am J Med $\mathbf{7 5}$ (1A):11-18.

McChesney EW, Banks WF Jr, and Fabian RJ (1967) Tissue distribution of chloroquine, hydroxychloroquine, and desethylchloroquine in the rat. Toxicol Appl Pharmacol 10:501-513.

Montcourrier P, Mangeat PH, Valembois C, Salazar G, Sahuquet A, Duperray C, and Rochefort $\mathrm{H}$ (1994) Characterization of very acidic phagosomes in breast cancer cells and their association with invasion. J Cell Sci 107:2381-2391.

Moore BR, Page-sharp M, Stoney JR, Ilett KF, Jago JD, Batty KT, and Al MET (2011) Pharmacokinetics, pharmacodynamics, and allometric scaling of chloroquine in a murine malaria model. Antimicrob Agents Chemother 55:3899-3907.

Ndolo RA, Luan Y, Duan S, Forrest ML, and Krise JP (2012) Lysosomotropic properties of weakly basic anticancer agents promote cancer cell selectivity in vitro. PLoS One 7:e49366. 
Palmieri M, Impey S, Kang H, di Ronza A, Pelz C, Sardiello M, and Ballabio A (2011) Characterization of the CLEAR network reveals an integrated control of cellular clearance pathways. Hum Mol Genet 20:3852-3866.

Pellegrini P, Strambi A, Zipoli C, Hägg-Olofsson M, Buoncervello M, Linder S, and De Milito A (2014) Acidic extracellular $\mathrm{pH}$ neutralizes the autophagyinhibiting activity of chloroquine: implications for cancer therapies. Autophagy 10: 562-571.

Persi E, Duran-Frigola M, Damaghi M, Roush WR, Aloy P, Cleveland JL, Gillies RJ, and Ruppin E (2018) Systems analysis of intracellular $\mathrm{pH}$ vulnerabilities for cancer therapy. Nat Commun 9:2997.

Ruzickova E, Skoupa N, Dolezel P, Smith DA, and Mlejnek P (2019) The lysosomal sequestration of tyrosine kinase inhibitors and drug resistance. Biomolecules $\mathbf{9}$ 675 .

Schmitt MV, Lienau P, Fricker G, and Reichel A (2019) Quantitation of lysosomal trapping of basic lipophilic compounds using in vitro assays and in silico predictions based on the determination of the full $\mathrm{pH}$ profile of the endo-/lysosomal system in rat hepatocytes. Drug Metab Dispos 47:49-57.

Settembre C, Di Malta C, Polito VA, Garcia Arencibia M, Vetrini F, Erdin S, Erdin SU, Huynh T, Medina D, Colella P, et al. (2011) TFEB links autophagy to lysosomal biogenesis. Science 332:1429-1433.

Settembre C, Zoncu R, Medina DL, Vetrini F, Erdin S, Erdin S, Huynh T, Ferron M, Karsenty G, Vellard MC, et al. (2012) A lysosome-to-nucleus signalling mechanism senses and regulates the lysosome via mTOR and TFEB. EMBO J 31:1095-1108.

Skoupa N, Dolezel P, and Mlejnek P (2020) Lysosomal fusion: an efficient mechanism increasing their sequestration capacity for weak base drugs without apparent lysosomal biogenesis. Biomolecules 10:1-37.

Tol MJ, van der Lienden MJC, Gabriel TL, Hagen JJ, Scheij S, Veenendaal T, Klumperman J, Donker-Koopman WE, Verhoeven AJ, Overkleeft H, et al. (2018) HEPES activates a MiT/TFE-dependent lysosomal-autophagic gene network in cultured cells: a call for caution. Autophagy 14:437-449.
Towers CG, Fitzwalter BE, Regan D, Goodspeed A, Morgan MJ, Liu CW, Gustafson DL, and Thorburn A (2019) Cancer cells upregulate NRF2 signaling to adapt to autophagy inhibition. Dev Cell 50:690-703.e6.

Trapp S, Rosania GR, Horobin RW, and Kornhuber J (2008) Quantitative modeling of selective lysosomal targeting for drug design. Eur Biophys $J$ 37:1317-1328.

Wang K, Tu Y, Wan J-B, Chen M, and He C (2019) Synergistic anti-breast cancer effect of pulsatilla saponin D and camptothecin through interrupting autophagiclysosomal function and promoting p62-mediated ubiquitinated protein aggregation. Carcinogenesis 41:804-816.

Wang L, Niu Z, Zhang L, Liu X, Wang X, Li F, and Wang Y (2012) Clinicopathological significance of mesothelin expression in invasive breast cancer. $J$ Int Med Res 40: 909-916.

Wei Y, Nygard GA, Ellertson SL, and Khalil SKW (1995) Stereoselective disposition of hydroxychloroquine and its metabolite in rats. Chirality 7:598-604.

Wojtkowiak JW, Verduzco D, Schramm KJ, and Gillies RJ (2011) Drug resistance and cellular adaptation to tumor acidic $\mathrm{pH}$ microenvironment. Mol Pharm 8 2032-2038.

Zhao B, Dierichs L, Gu JN, Trajkovic-Arsic M, Axel Hilger R, Savvatakis K, VegaRubin-de-Celis S, Liffers ST, Peña-Llopis S, Behrens D, et al. (2020) TFEBmediated lysosomal biogenesis and lysosomal drug sequestration confer resistance to MEK inhibition in pancreatic cancer. Cell Death Discov 6:12

Zhitomirsky B, Yunaev A, Kreiserman R, Kaplan A, Stark M, and Assaraf YG (2018) Lysosomotropic drugs activate TFEB via lysosomal membrane fluidization and consequent inhibition of mTORC1 activity. Cell Death Dis 9:1191.

Address correspondence to: Dr. Daniel L. Gustafson, Flint Animal Cancer Center, 300 West Drake Rd., FACC246, Fort Collins, CO 80523. E-mail: daniel.gustafson@colostate.edu 